DOI: https://doi.org/10.24867/02BE12Kundacina

\title{
MATEMATIČKI MODEL TRANSFORMATORA SA NAMOTAJIMA SPREGNUTIM U SLOMLjENU ZVEZDU
}

\section{MATHEMATICAL MODEL OF TRANSFORMER WITH ZIGZAG CONNECTED WINDINGS}

\author{
Ognjen Kundačina, Marko Obrenić, Predrag Vidović, Fakultet tehničkih nauka, Novi Sad
}

\section{Oblast - ELEKTROTEHNIKA I RAČUNARSTVO}

Kratak sadržaj - U okviru rada objašnjen je način modelovanja transformatora sa namotajima spregnutim u slomljenu zvezdu za potrebe osnovnih proračuna u elektroenergetskim sistemima. U teorijskom delu rada izvedene su generalne ekvivalentne šeme transformatora sprege Yzl, Yz5 i Yz9, a zatim su izvedene i konkretne ekvivalentne šeme transformatora sa spregom Yz5. U praktičnom delu rada izvršena je verifikacija izvedenih modela vršeći proračune tokova snaga za jednostavne test mreže.

Ključne reči: Transformatori, Konekcije namotaja transformatora, Slomljena zvezda

\begin{abstract}
This paper presents the method for modeling a power transformer with zigzag connected windings for the purpose of basic calculations in electrical power systems. In the theoretical part of the work, general equivalent schemes for $Y z 1, Y z 5$ and $Y z 9$ connected transformers are derived, and then specific equivalent schemes for $Y z 5$ transformer connection are derived. In the practical part of the work, verification of derived models is performed using the Load Flow calculations on simple test networks.
\end{abstract}

Keywords: Transformers, Transformer winding connections, Zigzag

\section{UVOD}

Transformatori su statički elektroenergetski uređaji pomoću kojih se vrši unutrašnja transformacija električne energije (u električnu energiju) [1]. Pri tome se menjaju napon i struja, sa težnjom da se snaga (energija) i napon kvalitativno ne degradiraju. Budući da su transformatori osnovni elementi elektroenergetskog sistema (EES), bitan zadatak je odrediti njihov matematički model za potrebe osnovnih proračuna u EES-u poput tokova snaga i kratkih spojeva.

Cilj ovog rada jeste da se izvedu ekvivalentne šeme trofaznih transformatora sa niženaponskim namotajem spregnutim u slomljenu zvezdu, u stacionarnom režimu, kao i da se iste numerički verifikuju na prostoj test mreži. Teorijska izvođenja koja se nalaze u ovom radu zasnovana su na neobjavljenom radu navedenom u pregledu literature [2]. Prema tome namotaj transformatora može biti spregnut u trougao, zvezdu i slomljenu zvezdu.

\section{NAPOMENA:}

Ovaj rad proistekao je iz master rada čiji mentor je bio doc. dr Predrag Vidović.
U trećoj glavi se izveden matematički model verifikuje pomoću računarskog programa napisanog u programskom jeziku $\mathrm{C}++$, koji vrši proračun tokova snaga metodom sumiranja struja na jednostavnim test mrežama [3].

\section{MATEMATIČKI MODEL TRANSFORMATORA SA SPREGOM Yz1, Yz5 i Yz9}

U ovoj glavi su prvo izvedene ekvivalentne šeme transformatora čiji je primarni namotaj spregnut u zvezdu, a sekundarni u slomljenu zvezdu, na taj način da sprežni broj iznosi 1, 5 ili 9. Zatim su izvedene konkretne ekvivalentne šeme za transformator sa spregom Yz5.

\subsection{Sprege Yz1, Yz5 i Yz9}

U svrhu izvođenja ekvivalentnih šema razmatra se trofazni (trostubni, četvorostubni ili) petostubni, uravnotežen transformator prikazan na slici 1 . On je doveden $u$ stacionaran režim. Brojevi navojaka (N) i otpornosti namotaja $(\mathrm{R})$ svakog od trojke namotaja na istoj strani transformatora jednaki su međusobno, pa nisu označeni uz svaki namotaj. Treba napomenuti da se niženaponski (NN) namotaj, koji je spregnut u slomljenu zvezdu, sastoji od dva polunamotaja smeštena na različite stubove. Transformator se sastoji od tri namotaja po fazi na višenaponskoj (VN) strani: 1-1', 3-3' i 5-5', i šest namotaja na NN strani (po dva električno povezana): 2-2', 4-4' i 6-6', koji su međusobno magnetski spregnuti. Električne veličine koje karakterišu režim transformatora su: naponi $u_{1}, u_{2}, u_{3}, u_{4}, u_{5}$ i $u_{6}$ i struje $i_{1}, i_{2}, i_{3}, i_{4}, i_{5}$ i $i_{6}$. U magnetske veličine spadaju: fluksevi rasipanja $\phi_{\mathrm{I} \gamma 1}, \phi_{\mathrm{I} \gamma 21}, \phi_{\mathrm{I} \gamma 22}, \phi_{\mathrm{II} \gamma 1}, \phi_{\mathrm{II} \gamma 21}, \phi_{\mathrm{II} \gamma 22}, \phi$ ${ }_{\text {III } 1}, \phi_{\text {III } 21}$ i $\phi_{\text {III } 22}$ i zajednički fluksevi po svakom stubu transformatora $\phi_{\text {I }}, \phi_{\text {II }}$ i $\phi_{\text {III }}$, kao i njihov zbir $\phi$. Nesimetričan režim razmatranog uravnoteženog transformatora se može prikazati s tri jednofazna reprezenta direktnog (d), inverznog (i) i nultog redosleda (o).

Svakom od njih se asocira po jedna pogonska (jednofazna) ekvivalentna šema, koje su međusobno raspregnute. Svaka od njih se može izvesti razmatranjem trofaznog transformatora $u$ simetričnom režimu odgovarajućeg redosleda. To se može učiniti "nametanjem" trofaznih simetričnih napona (izabrane vrste simetrije) na oba kraja transformatora, čime je i trofazni režim uravnoteženog transformatora simetričan iste vrste simetrije kao i pobuda. U simetričnim režimima direktnog i inverznog redosleda, suma flukseva prvog, drugog i trećeg stuba transformatora jednaka je nuli. Međutim, u simetričnom režimu nultog redosleda, fluksevi tih stubova međusobno su u fazi, pa je njihov zbir jednak trostrukoj vrednosti fluksa jednog stuba. 


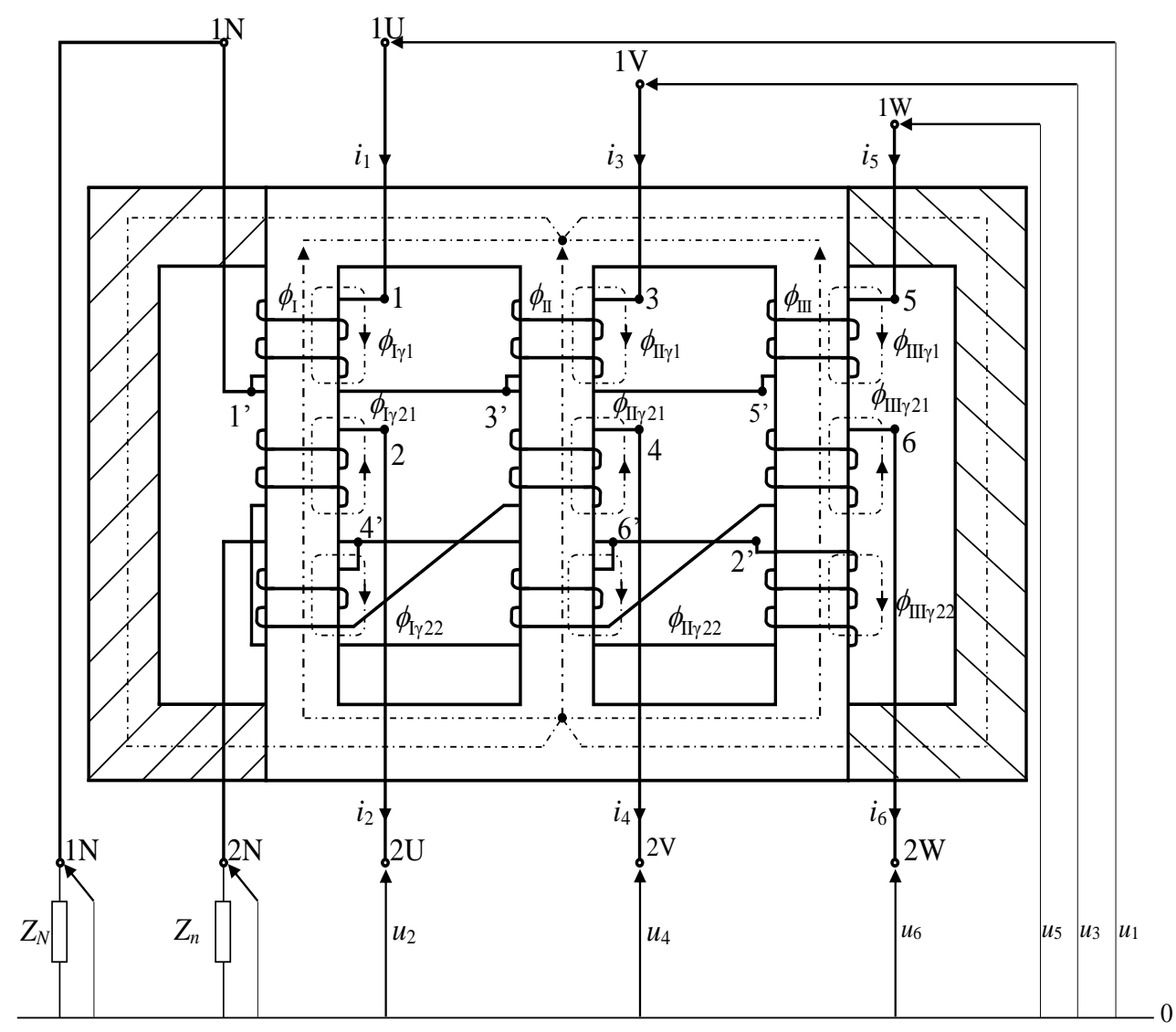

Slika 1. Principska šema trofaznog, dvonamotajnog transformatora sprege Yz1, Yz5, Yz9

Matematički opis režima tri namotaja, u simetričnom režimu sastoji se od dve relacije saglasne s naponskim Kirhofovim zakonom (drugi i treći namotaj su električno spojeni pa čine jednu konturu), četiri relacije za četiri magnetne konture, kao i od jedne relacije s kojom se izražava kontinuitet flukseva u transformatoru:

$$
\begin{aligned}
& u_{1}= R_{1} i_{1}+N_{1} \frac{d \phi_{\mathrm{I} \gamma 1}}{d t}+N_{1} \frac{d \phi_{\mathrm{I}}}{d t}, \\
& u_{2}=-\frac{R_{2}}{2} i_{2}-\frac{R_{2}}{2} i_{2}+\frac{N_{2}}{2} \frac{d \phi_{\mathrm{I}}}{d t}-\frac{N_{2}}{2} \frac{d \phi_{\mathrm{III}}}{d t} \\
&-\frac{N_{2}}{2} \frac{d \phi_{\mathrm{I} \gamma 21}}{d t}-\frac{N_{2}}{2} \frac{d \phi_{\mathrm{III} \gamma 22}}{d t}, \\
& N_{1} i_{1}-\frac{N_{2}}{2} i_{2}+\frac{N_{2}}{2} i_{4}=\mathfrak{R}_{\mathrm{s}} \phi_{\mathrm{I}}+\alpha_{\mathrm{Fe}} \frac{d \phi_{\mathrm{I}}}{d t}+\mathfrak{R}_{\mathrm{u}} \phi+\alpha_{\mathrm{u}} \frac{d \phi}{d t}, \\
& N_{1} i_{1}= \mathfrak{R}_{\mathrm{I} \gamma 1} \phi_{\mathrm{I} \gamma 1}, \\
& \frac{N_{2}}{2} i_{2}=\mathfrak{R}_{\mathrm{I} \gamma 21} \phi_{\mathrm{I} \gamma 21}, \\
& \frac{N_{2}}{2} i_{4}=\mathfrak{R}_{\mathrm{I} \gamma 22} \phi_{\mathrm{I} \gamma 22}, \\
& \phi=\phi_{\mathrm{I}}+\phi_{\mathrm{II}}+\phi_{\mathrm{III}},
\end{aligned}
$$

gde su $\mathfrak{R}_{\gamma}$ magnetni otpori kontura rasipnih flukseva, $\mathfrak{R}_{\mathrm{s}}$ magnetni otpori stubova, $\Re_{u}$ magnetni otpor "povratnog puta" fluksa (četvrtog i petog stuba, odnosno okoline), $\alpha_{\mathrm{Fe}}$ magnetna karakteristika gvožđa kojom se modeluje aktivna snaga gubitaka u gvožđu (magnetnom kolu) i $\alpha_{\mathrm{u}}$ magnetna karakteristika sredine.

Prelaskom iz vremenskog u kompleksni domen, zamenom proizvoda tipa $\omega N^{2} / \mathfrak{R}$, dobijenih uvrštavanjem izraza za flukseve rasipanja u električni deo modela za par namotaja na istom stubu, dobija se:

$\hat{U}_{1}=\left(R_{1}+\mathrm{j} X_{\gamma 1}\right) \hat{I}_{1}+N_{1} \hat{E}_{\mathrm{I}}$,

$\hat{U}_{2}=-\left(R_{2}+\mathrm{j} X_{\gamma 2}\right) \hat{I}_{2}+\frac{N_{2}}{2} \hat{E}_{\mathrm{I}}-\frac{N_{2}}{2} \hat{E}_{\mathrm{III}}$,

$\hat{I}_{\mathrm{m}}^{1}=\hat{I}_{1}-\frac{N_{2}}{2 N_{1}} \hat{I}_{2}+\frac{N_{2}}{2 N_{1}} \hat{I}_{4}$,

$N_{1} \hat{E}_{\mathrm{I}}=\hat{Z}_{\mathrm{m}}^{1} \hat{I}_{\mathrm{m}}^{1}$

gde je je impedansa magnećenja svedena na stranu 1 , za namotaje na istom stubu:

$\hat{Z}_{\mathrm{m}}^{1}=\frac{\omega^{2}\left(N_{1}\right)^{2} \alpha_{\mathrm{m}}}{\left(\Re_{\mathrm{m}}\right)^{2}+\omega^{2}\left(\alpha_{\mathrm{m}}\right)^{2}}+\mathrm{j} \frac{\omega\left(N_{1}\right)^{2} \Re_{\mathrm{m}}}{\left(\Re_{\mathrm{m}}\right)^{2}+\omega^{2}\left(\alpha_{\mathrm{m}}\right)^{2}}=R_{\mathrm{m}}^{1}+\mathrm{j} X_{\mathrm{m}}^{1}$.

Shodno vezama između elektromotornih sila na različitim stubovima transformatora i struja namotaja, model transformatora za simetrične režime direktnog, inverznog i nultog redosleda predstavljen je relacijama 4,5 i 6.

Ekvivalentna šema, kojom se opisuje režim namotaja trofaznog transformatora, ima oblik prikazan na slici 2 , a oznake korištene na slici su definisane relacijama 7 . 


$$
\begin{aligned}
& \hat{U}_{1}^{\mathrm{d}}=\left(R_{1}+\mathrm{j} X_{\gamma 1}\right) \hat{I}_{1}^{\mathrm{d}}+N_{1} \hat{E}_{\mathrm{I}}^{\mathrm{d}}, \\
& \hat{U}_{2}^{\mathrm{d}}=-\left(R_{2}+\mathrm{j} X_{\gamma 2}\right) \hat{I}_{2}^{\mathrm{d}}+\sqrt{3} \frac{N_{2}}{2} \mathrm{e}^{-\mathrm{j} \pi / 6} \hat{E}_{\mathrm{I}}^{\mathrm{d}} \text {, } \\
& \hat{I}_{\mathrm{m}}^{\mathrm{ld}}=\hat{I}_{1}^{\mathrm{d}}-\sqrt{3} \frac{N_{2}}{2 N_{1}} \mathrm{e}^{\mathrm{j} \pi / 6} \hat{I}_{2}^{\mathrm{d}}, \\
& N_{1} \hat{E}_{\mathrm{I}}^{\mathrm{d}}=\hat{Z}_{\mathrm{m}}^{1 \mathrm{~d}} \hat{I}_{\mathrm{m} 2}^{1 \mathrm{~d}} \\
& \hat{U}_{1}^{\mathrm{i}}=\left(R_{1}+\mathrm{j} X_{\gamma 1}\right) \hat{I}_{1}^{\mathrm{i}}+N_{1} \hat{E}_{\mathrm{I}}^{\mathrm{i}} \text {, } \\
& \hat{U}_{2}^{\mathrm{i}}=-\left(R_{2}+\mathrm{j} X_{\gamma 2}\right) \hat{I}_{2}^{\mathrm{i}}+\sqrt{3} \frac{N_{2}}{2} \mathrm{e}^{\mathrm{j} \pi / 6} \hat{E}_{\mathrm{I}}^{\mathrm{i}}, \\
& \hat{I}_{\mathrm{m}}^{\mathrm{li}}=\hat{I}_{1}^{\mathrm{i}}-\sqrt{3} \frac{N_{2}}{2 N_{1}} \mathrm{e}^{-\mathrm{j} \pi / 6} \hat{I}_{2}^{\mathrm{i}}, \\
& N_{1} \hat{E}_{\mathrm{I}}^{\mathrm{i}}=\hat{Z}_{\mathrm{m}}^{1 \mathrm{i}} \hat{I}_{\mathrm{m} 2}^{\mathrm{i}} \\
& \hat{U}_{1}^{\mathrm{o}}=\left(R_{1}+\mathrm{j} X_{\gamma 1}\right) \hat{I}_{1}^{\mathrm{o}}+N_{1} \hat{E}_{\mathrm{I}}^{\mathrm{o}}, \\
& \hat{U}_{2}^{\mathrm{o}}=-\left(R_{2}+\mathrm{j} X_{\gamma 2}\right) \hat{I}_{2}^{\mathrm{o}} \text {, } \\
& \hat{I}_{\mathrm{m}}^{\mathrm{lo}}=\hat{I}_{1}^{\mathrm{o}} \text {, } \\
& N_{1} \hat{E}_{\mathrm{I}}^{\mathrm{o}}=\hat{Z}_{\mathrm{m}}^{1 \mathrm{o}} \hat{I}_{\mathrm{m}}^{\mathrm{lo}} \\
& \hat{Z}_{1}=R_{1}+\mathrm{j} X_{\gamma 1} \text {, } \\
& \hat{Z}_{2}=R_{2}+\mathrm{j} X_{\gamma 2}, \\
& N_{1} \hat{E}_{\mathrm{I}}^{\mathrm{x}}=\hat{E}_{1}^{\mathrm{x}}, \quad x=\mathrm{d}, \mathrm{i}, \mathrm{o}, \\
& \sqrt{3} \frac{N_{2}}{2} \mathrm{e}^{-\mathrm{j} k^{x} \pi / 6} \hat{E}_{\mathrm{I}}^{x}=\hat{E}_{2}^{x}, \quad x=\mathrm{d}, \mathrm{i}, \\
& \hat{I}_{\mathrm{m}}^{1 x}=\hat{I}_{1}^{x}-\sqrt{3} \frac{N_{2}}{2 N_{1}} \mathrm{e}^{\mathrm{j} k^{x} \pi / 6} \hat{I}_{2}^{x}, \quad x=\mathrm{d}, \mathrm{i} .
\end{aligned}
$$

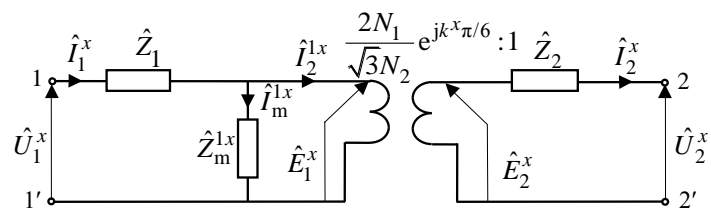

(a)

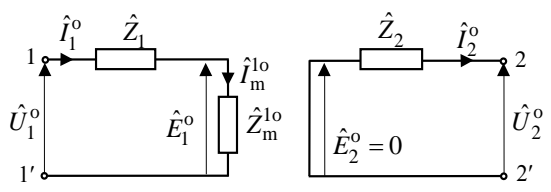

(b)

Slika 2. Ekvivalentna šema trofaznog transformatora sprege $Y z 1, Y z 5$ i $Y z$ 9; a-za simetričan režim direktnog $(x=d)$, inverznog $(x=i) ; b-$ nultog redosleda

\subsection{Sprega Yz5}

Da bi se realizovala sprega Yz5, transformator sa slike 1 (njegovi priključci - $1 \mathrm{U}, 1 \mathrm{~V}, 1 \mathrm{~W}$ i $1 \mathrm{~N}$, odnosno $2 \mathrm{~W}, 2 \mathrm{U}$, $2 \mathrm{~V}$ i $2 \mathrm{~N}$ ) se priključuju na elektroenergetski sistem na faze A, B i C, odnosno c, a i b (VN i NN strana, respektivno). Transformator se nalazi u okviru elektroenergetskog sistema, da bi se sagledao i s aspekta simetričnih režima nultog redosleda. U tim režimima struje po fazama čine konture kojima su obuhvaćene i veze transformatora sa zemljom, kao i sama zemlja. Neka se razmatrani transformator nalazi u simetričnom režimu bilo koje vrste simetrije „,X“. Tada su režimi svakog stuba, sa svojim parom namotaja, isti, sa odgovarajućim pomerajem u vremenu (zavisnim od vrste simetrije razmatranog režima). Kod ovog transformatora između tačaka 1-1' priključen je napon faze A, a između tačaka 22 ' napon faze $\mathrm{c}$. Pošto je suma faznih struja impedansi uzemljenja, na VN i NN strani transformatora, u simetričnim režimima direktnog i inverznog redosleda jednaka nuli i naponi, $\hat{U}_{0 \mathrm{~N}}^{x}$ i $\hat{U}_{0 \mathrm{n}}^{x}, x=\mathrm{d}, \mathrm{i}$, takođe su jednaki nuli, te tačke $\mathrm{N}, \mathrm{n}$ i 0 predstavljaju istu tačku. Tako se šema razmatranog transformatora, za simetrične režime direktnog $(\mathrm{x}=\mathrm{d}) \mathrm{i}$ inverznog redosleda $(\mathrm{x}=\mathrm{i})$, može prikazati kao na slici 3 .

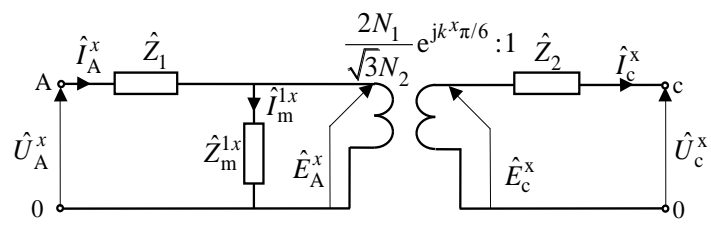

(a)

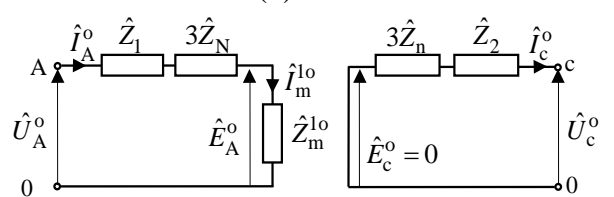

(b)

Slika 3. Ekvivalentna šema trofaznog transformatora sprege $Y z 5 ; a-z$ a simetričan režim direktnog $(x=d$; $\left.k^{d}=5\right)$, inverznog $\left(x=i ; k^{i}=7\right) ; b$-nultog redosleda

$\mathrm{Za}$ simetrične režime direktnog, inverznog i nultog redosleda važe sledeće relacije:

$$
\begin{aligned}
& \hat{E}_{\mathrm{c}}^{\mathrm{d}}=\hat{E}_{\mathrm{a}}^{\mathrm{d}} \mathrm{e}^{\mathrm{j} 2 \pi / 3}, \\
& \hat{U}_{\mathrm{c}}^{\mathrm{d}}=\hat{U}_{\mathrm{a}}^{\mathrm{d}} \mathrm{e}^{\mathrm{j} 2 \pi / 3}, \\
& \hat{I}_{\mathrm{c}}^{\mathrm{d}}=\hat{I}_{\mathrm{a}}^{\mathrm{d}} \mathrm{e}^{\mathrm{j} 2 \pi / 3}, \\
& \hline \hat{E}_{\mathrm{c}}^{\mathrm{i}}=\hat{E}_{\mathrm{a}}^{\mathrm{i}} \mathrm{e}^{\mathrm{j} \pi \pi / 3}, \\
& \hat{U}_{\mathrm{c}}^{\mathrm{i}}=\hat{U}_{\mathrm{a}}^{\mathrm{i}} \mathrm{e}^{\mathrm{j} \pi \pi / 3}, \\
& \hat{I}_{\mathrm{c}}^{\mathrm{i}}=\hat{I}_{\mathrm{a}}^{\mathrm{i}} \mathrm{e}^{\mathrm{j} 4 \pi / 3}, \\
& \hat{E}_{\mathrm{c}}^{\mathrm{o}}=\hat{E}_{\mathrm{a}}^{\mathrm{o}}, \\
& \hat{U}_{\mathrm{c}}^{\mathrm{o}}=\hat{U}_{\mathrm{a}}^{\mathrm{o}}, \\
& \hat{I}_{\mathrm{c}}^{\mathrm{o}}=\hat{I}_{\mathrm{a}}^{\mathrm{o}} .
\end{aligned}
$$

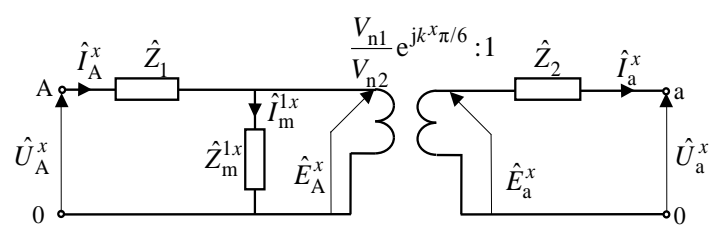

(a)

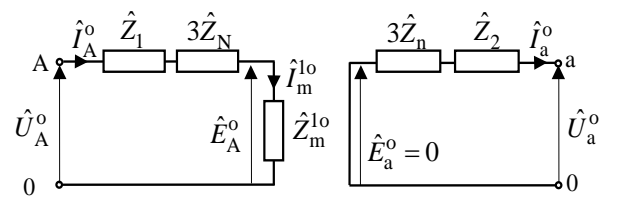

(b)

Slika 4. Pogonska šema trofaznog transformatora sprege $Y z 5 ; a-z a$ simetričan režim direktnog $\left(x=d ; k^{d}=5\right)$, inverznog $\left(x=i ; k^{i}=7\right) ; b-$ nultog redosleda 
Relacije 8, 9 i 10 omogućavaju da se ekvivalentne šeme razmatranog trofaznog transformatora, za simetričan režim direktnog, inverznog i nultog redosleda, prikažu kao na slici 4. Šeme na slici 4a i b, definitivno jesu pogonske. Njima su povezani samo fazni naponi i struje iste faze sa obe strane transformatora.

\section{NUMERIČKA VERIFIKACIJA IZVEDENOG MATEMATIČKOG MODELA}

U okviru ove glave je na primeru jednostavnih test mreža verifikovan matematički model transformatora sa namotajima spregnutim u slomljenu zvezdu. Model se verifikuje proračunom tokova snaga $u$ domenu simetričnih komponenti i relativnih vrednosti. Test mreže, koje su prikazane na slikama 5 i 6 , se razlikuju samo po sprezi NN namotaja transformatora. U prvoj test mreži NN namotaj transformatora je spregnut $u$ (direktno uzemljenu) slomljenu zvezdu, a u drugoj u (direktno uzemljenu) zvezdu.

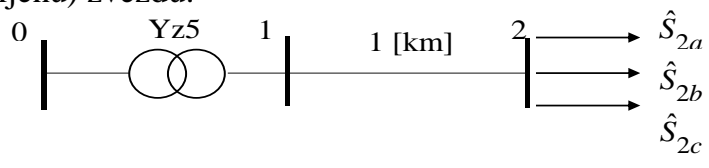

Slika 5. Test mreža 1

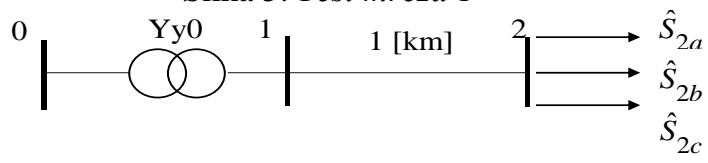

Slika 6. Test mreža 2

Potrošnja u čvoru 2 za obe test mreže iznosi: $\hat{S}_{2 a}=(30$ -

j15) kVA, $\hat{S}_{2 b}=(20-\mathrm{j} 10) \mathrm{kVA}, \hat{S}_{2 c}=(10-\mathrm{j} 5) \mathrm{kVA}$.

Naponi čvora 0 iznose: $\hat{U}^{\mathrm{d}}=5.774 \mathrm{e}^{\mathrm{j} 0.0^{\circ}} \mathrm{kV}, \hat{U}^{\mathrm{i}}=$ $1.020 \mathrm{e}^{\mathrm{j} 0.0^{\circ}} \mathrm{kV}, \quad \hat{U}^{\mathrm{o}}=1.020 \mathrm{e}^{\mathrm{j} 0.0^{\circ}} \mathrm{kV}$. Simetrične komponente napona čvorova test mreža 1 i 2 , u domenu relativnih vrednosti, prikazane su u tabelama 1 i 2 .

Tabela 1. Naponi čvorova test mreže 1

\begin{tabular}{|c|c|c|c|}
\hline Čvor & $\hat{U}^{\mathrm{d}}$ & $\hat{U}^{\mathrm{i}}$ & $\hat{U}^{\mathrm{o}}$ \\
\hline 0 & $1.000 \mathrm{e}^{\mathrm{j} 0.0^{\circ}}$ & $0.177 \mathrm{e}^{\mathrm{j} 0.0^{\circ}}$ & $0.177 \mathrm{e}^{\mathrm{j} 0.0^{\circ}}$ \\
\hline 1 & $0.985 \mathrm{e}^{-\mathrm{j} 150.609^{\circ}}$ & $0.180 \mathrm{e}^{\mathrm{j} 148.972^{\circ}}$ & $0.003 \mathrm{e}^{\mathrm{j} 3.447^{\circ}}$ \\
\hline 2 & $0.970 \mathrm{e}^{-\mathrm{j} 150.852^{\circ}}$ & $0.182 \mathrm{e}^{\mathrm{j} 147.816^{\circ}}$ & $0.020 \mathrm{e}^{\mathrm{j} 12.855^{\circ}}$ \\
\hline
\end{tabular}

Tabela 2. Naponi čvorova test mreže 2

\begin{tabular}{|c|c|c|c|}
\hline Čvor & $\hat{U}^{\mathrm{d}}$ & $\hat{U}^{\mathrm{i}}$ & $\hat{U}^{\mathrm{o}}$ \\
\hline 0 & $1.000 \mathrm{e}^{\mathrm{j} 0.0^{\circ}}$ & $0.177 \mathrm{e}^{\mathrm{j} 0.0^{\circ}}$ & $0.177 \mathrm{e}^{\mathrm{j} 0.0^{\circ}}$ \\
\hline 1 & $0.986 \mathrm{e}^{\mathrm{-j} 0.626^{\circ}}$ & $0.178 \mathrm{e}^{-\mathrm{j} 1.150^{\circ}}$ & $0.174 \mathrm{e}^{\mathrm{j} 0.670^{\circ}}$ \\
\hline 2 & $0.971 \mathrm{e}^{\mathrm{j} 0.898^{\circ}}$ & $0.178 \mathrm{e}^{-\mathrm{j} 2.146^{\circ}}$ & $0.169 \mathrm{e}^{\mathrm{j} 3.361^{\circ}}$ \\
\hline
\end{tabular}

Nulta komponenta napona na primaru transformatora sa spregom Yz se ne prenosi na sekundar transformatora, za razliku od transformatora sa spregom Yy. U tabelama $3 \mathrm{i}$ 4 prikazane su vrednosti struja grana test mreža 1 i 2 , takođe izraženih $\mathrm{u}$ domenu simetričnih komponenti i relativnih vrednosti. Nulta komponenta struje koja teče kroz sekundarni namotaj transformatora sa spregom Yz se ne prenosi na primarni namotaj transformatora, za razliku od transformatora sa spregom Yy.
Tabela 3. Struje grana test mreže 1

\begin{tabular}{|c|c|c|c|}
\hline Grana & $\hat{I}^{\mathrm{d}}$ & $\hat{I}^{\mathrm{i}}$ & $\hat{I}^{\mathrm{o}}$ \\
\hline $0-1$ & $0.234 \mathrm{e}^{-\mathrm{j} 30.259^{\circ}}$ & $0.059 \mathrm{e}^{\mathrm{j} 70.792^{\circ}}$ & $0.001 \mathrm{e}^{-\mathrm{j} 80.401^{\circ}}$ \\
\hline $1-2$ & $0.234 \mathrm{e}^{-\mathrm{j} 179.740^{\circ}}$ & $0.059 \mathrm{e}^{-\mathrm{j} 139.208^{\circ}}$ & $0.078 \mathrm{e}^{\mathrm{j} 119.232^{\circ}}$ \\
\hline
\end{tabular}

Tabela 4. Struje grana test mreže 2

\begin{tabular}{|c|c|c|c|}
\hline Grana & $\hat{I}^{\mathrm{d}}$ & $\hat{I}^{\mathrm{i}}$ & $\hat{I}^{\mathrm{o}}$ \\
\hline $0-1$ & $0.226 \mathrm{e}^{\mathrm{j} 27.651^{\circ}}$ & $0.046 \mathrm{e}^{\mathrm{j} 42.537^{\circ}}$ & $0.043 \mathrm{e}^{-\mathrm{j} 100.106^{\circ}}$ \\
\hline $1-2$ & $0.226 \mathrm{e}^{\mathrm{j} 27.651^{\circ}}$ & $0.046 \mathrm{e}^{\mathrm{j} 42.537^{\circ}}$ & $0.043 \mathrm{e}^{-\mathrm{j} 100.106^{\circ}}$ \\
\hline
\end{tabular}

\section{ZAKLJUČAK}

U okviru ovog rada prikazan je model transforatora sa NN namotajem spregnutim u slomljenu zvezdu. U rezultatima verifikacionog proračuna tokova snaga za dve test mreže ističu se razlike u simetričnim režimima nultog redosleda dva transformatora, sprega Yz5 i Yy0. Kao posledica prekida u pogonskoj šemi za nulti redosled kod transformatora sprege Yz5, nulta komponenta napona se ne prenosi sa VN na NN stranu transformatora, a nulta komponenta struje se ne prenosi sa NN na VN stranu, što nije slučaj kod transformatora sprege $\mathrm{YyO}$.

\section{LITERATURA}

[1] V.C.Strezoski, S.R.Milakovic, "Ekvivalentne šeme elektroenergetskih transformatora u analizi stacionarnih režima”, Srbija, Novi Sad, Fakultet tehničkih nauka Novi Sad, EPS-JP "Elektrovojvodina", MP "STYLOS”, 1998.

[2] P.Vidović, "Transformatori sa niskonaponskim namotajima povezanim u slomljenu zvezdu", skripta, Fakultet tehničkih nauka, Novi Sad, 2018.

[3] D. Popović, D. Bekut, V. Treskanica, "Specijalizovani DMS algoritmi”, Srbija: Novi Sad, DMS Grupa DOO za elektroenergetski inžinjering, 2004.

\section{Kratka biografija:}

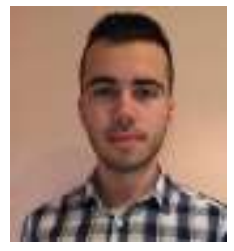

Ognjen Kundačina rođen je u Nevesinju, Bosna i Hercegovina, 1994. god. Osnovne studije završio je na Fakultetu tehničkih nauka iz oblasti Elektrotehnike i računarstva Elektroenergetski sistemi 2017. god. i 2017. god. upisao master studije na istom fakultetu. kontakt: ognjenkundacina94@gmail.com

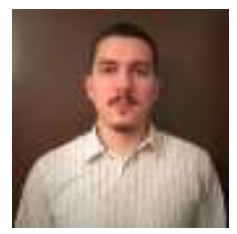

Marko Obrenić rođen je u Beogradu, Srbija, 1990. god. Osnovne i master studije zavrsio je na Fakultetu tehničkih nauka iz oblasti Elektrotehnike i računarstva Elektroenergetski sistemi 2013. i 2014. god. i 2014. god. upisao doktorske studije na istom fakultetu.

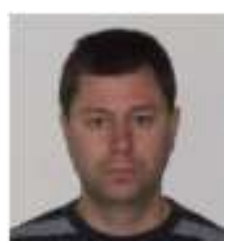

Predrag Vidović rođen u Vlasenici, Bosna i Hercegovina, 1981. god. Diplomirao je, magistrirao i doktorirao na Fakultetu tehničkih nauka iz oblasti Elektrotehnike i računarstva - Elektroenergetski sistemi 2005, 2008. i 2015. god, respektivno. 